\title{
Comparación estadística entre valores terrestres y satelitales de la velocidad media anual del viento
}

Ing. Daniel Nazario'; Ing. Valentín Stefanini²; Alumno Brandon Ortiz ${ }^{3}$

\section{RESUMEN}

Este trabajo se realiza con el objeto de aplicar métodos estadísticos para diferen-ciar el error que se comete cuando se usan estaciones meteorológicas terrestres o estacio-nes meteorológicas satelitales; tratando de encontrar la diferencia de medidas entre am-bas, y llegar a una conclusión válida para aplicar dichos resultados en la confección de mapas eólicos con orografía y rugosidad iguales, usando como base los ya realizados para la provincia de San Luis.

Palabras clave: Estadística, Errores, Estaciones Meteorológicas, Terrestres/Satelitales.

\section{ABSTRACT}

This work is carried out in order to apply statistical methods to differentiate the error that is committed when using terrestrial meteorological stations or sate- llite meteorological stations; trying to find the difference of measurements between both, and reach a valid conclusion to apply these results in the preparation of wind maps with equal orography and roughness, using as a base those already made for the province of San Luis.

Keywords: Statistics, Errors, Meteorological, Terrestrial / Satellite Stations.

\section{INTRODUCCIÓN:}

En el año 2011 se realizó el mapa eólico de la Provincia de San Luis, utilizando como herramienta el software Argentino Map (Open Wind), tomando los datos de esta-ciones meteorológicas terrestres propias, estaciones meteorológicas instaladas por la Universidad Provincial de la Punta, aeropuerto de Villa Reynolds y el aeropuerto de la ciudad de San Luis. Tiempo después de la elaboración del Mapa Eó-

\footnotetext{
${ }^{1}$ Ingeniero Daniel Nazario. Universidad Nacional de Villa Mercedes-Argentina. elmundial@hotmail.com ${ }^{2}$ Ingeniero Valentín Stefanini, Universidad Nacional de Villa Mercedes-Argentina. stefani-ni28@hotmail.com.ar ${ }^{3}$ Alumno Brandon Ortiz Universidad Nacional de Villa Mercedes-Argentina. elmundial@hotmail.com
} 
lico, se pudo contar con datos de las 56 estaciones meteorológicas terrestres REM (Red de Estaciones Me-teorológicas de la Provincia de San Luis),y además se pudo tener acceso a estaciones virtuales satelitales entre ellas la llamada MERRA2 ${ }^{1}$.

Utilizando los datos de vientos y direcciones de la REM, así como los datos obtenidos satelitalmente, para un mismo punto geográfico, se realizó con ellos, dos mapas eólicos de la Provincia de San Luis, con la orografía y la rugosidad iguales en ambos.

\section{METODOLOGIA}

Para el presente análisis de este trabajo, además de la asistencia a diferentes encuen-tros, cursos, foros nacionales e internacionales, hicieron posible la obtención de direcciones web que nos permitieron tener acceso a estaciones virtuales satelitales entre ellas la llamada MERRA2.

Como así también se contó con los aportes teóricos impartido por HECTOR FERNANDO MATIOS perteneciente al C.R.E.E Centro Regional Energía Eólica.

\section{MATERIALES}

Para la confección del presente trabajo se utilizaron varios softwares específicos del tema eolio como también del tema estadístico.

Entre ellos:

Software eólico: Open Wind, WINDOGRAPHER, Global MapperV17,

Software de procesamiento de datos SIG (Sistema de Información Geográfica) con todo tipo de información cartográfica y de mapas para profesionales.

Software de estadística IBM-SPSS Estadística y "R" libre

También se utilizaron datos de estaciones meteorológicas de la provincia de San Luis, y datos satelitales.

\section{DESARROLLO}

De la figura 1 , se observa que, para un mismo punto geográfico de ambos mapas, el valor difiere en su valor medio, por lo que el objetivo que se plantea en este trabajo, es obtener la relación entre el error porcentual existente en cada punto geográfico con respecto a los 2 mapas teniendo como datos: altura, orografía y rugosidad.

\footnotetext{
${ }^{1} 1$ Analisis retrospectivo moderno para investigación y aplicaciones. Descripción del pro-yecto. El análisis retrospectivo moderno para la investigación y aplicaciones, es la versión 2 (MERRA-2) comenzando a proporcionar datos desde 1980 esta versión 2 reemplaza al conjunto de datos MERRA Original debido al avance realizado en el sistema que permite la asimilación del resplandor hiperespectral moderno y observación de microondas, junto con los conjuntos de datos de ocultación GPS-Radio. Avances adicionales tanto en el mo-delo GEO como en el sistema de GIS que están incluidos en MERRA-2. Junto con las me-joras meteorológicas, MERRA-2 da algunos pasos significativos hacia el objetivo de GMAO de un nuevo análisis de sistema de la tierra. MERRA-2 es la primera reanálisis global a largo plazo para asimilar las observaciones espaciales
} 
1)

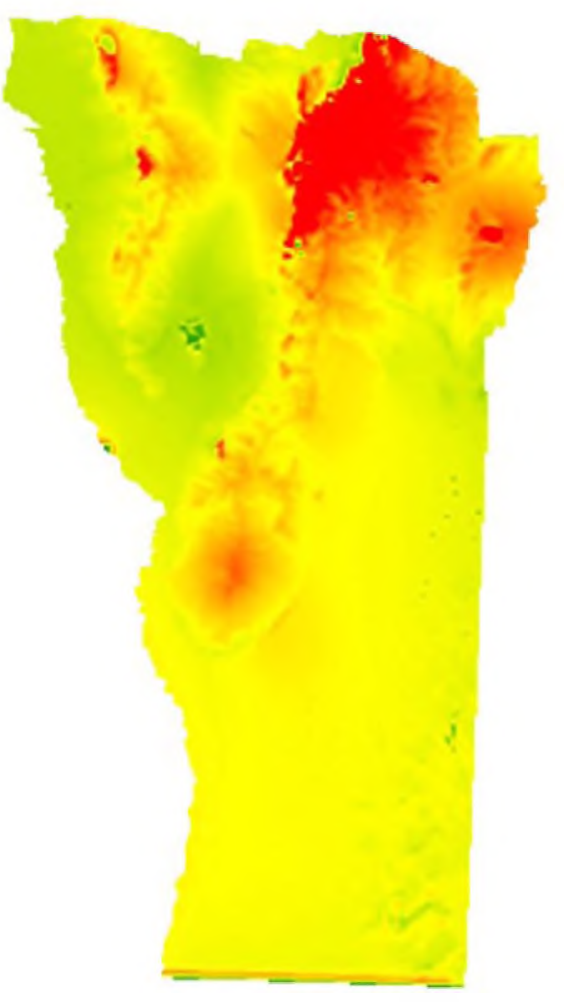

2)

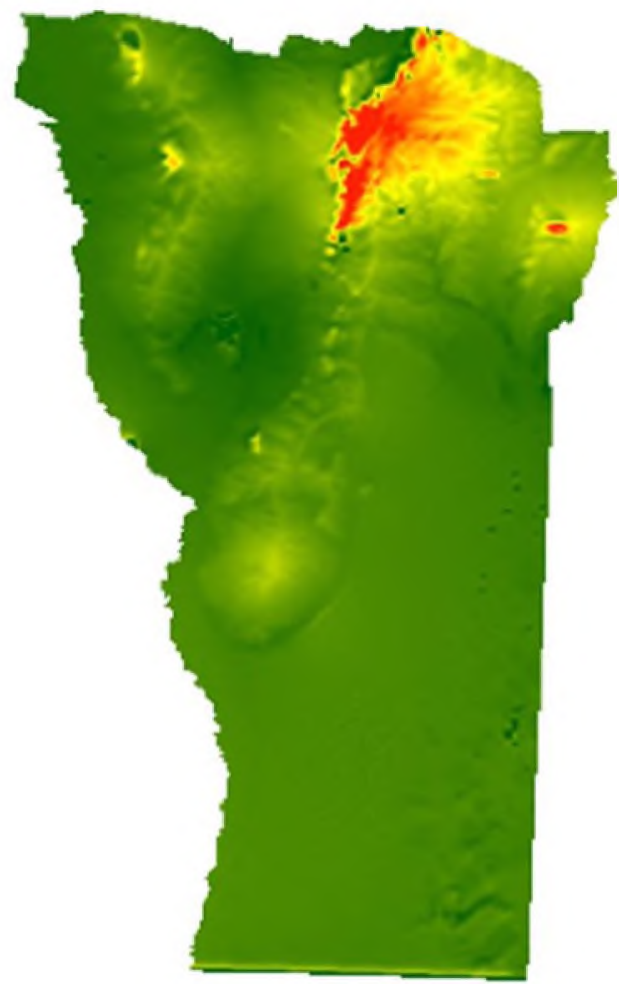

Figura 1: Mapa de vientos con: 1) estaciones meteorológicas terrestres, 2) $M E R R A-2$

La potencia por unidad de área perpendicular a la dirección del viento es:

$$
P=1 / 2 \rho V^{3} A
$$

Donde:

P: Potencia por unidad de área perpendicular a la dirección del viento Watt

$\rho:$ Densidad del aire $\mathrm{kg} / \mathrm{m} 3$

$\mathrm{V}$ : velocidad del viento $\mathrm{m} / \mathrm{seg}$.

A: área perpendicular $\mathrm{m} 2$

Para el análisis de los errores medios, tal cual se planteará anteriormente, se decidió la utilización del Software estadístico IBM-SPSS (en su versión demo, que es perfectamente operativa para el objetivo de este trabajo).

Con una simple observación visual de las Figuras 1 y 2, se puede notar la diferencia entre los valores de cada uno de los mapas de vientos en cuanto a sus valores, evidenciados por la colorimetría de ambos.

En el presente análisis de los datos de velocidad y dirección de los vientos, se toma como base los valores medios anuales con un vasto historial (18 años), observándose que el porcentaje de error es similar aproximado del 11,7\% (+-), en varios puntos. 


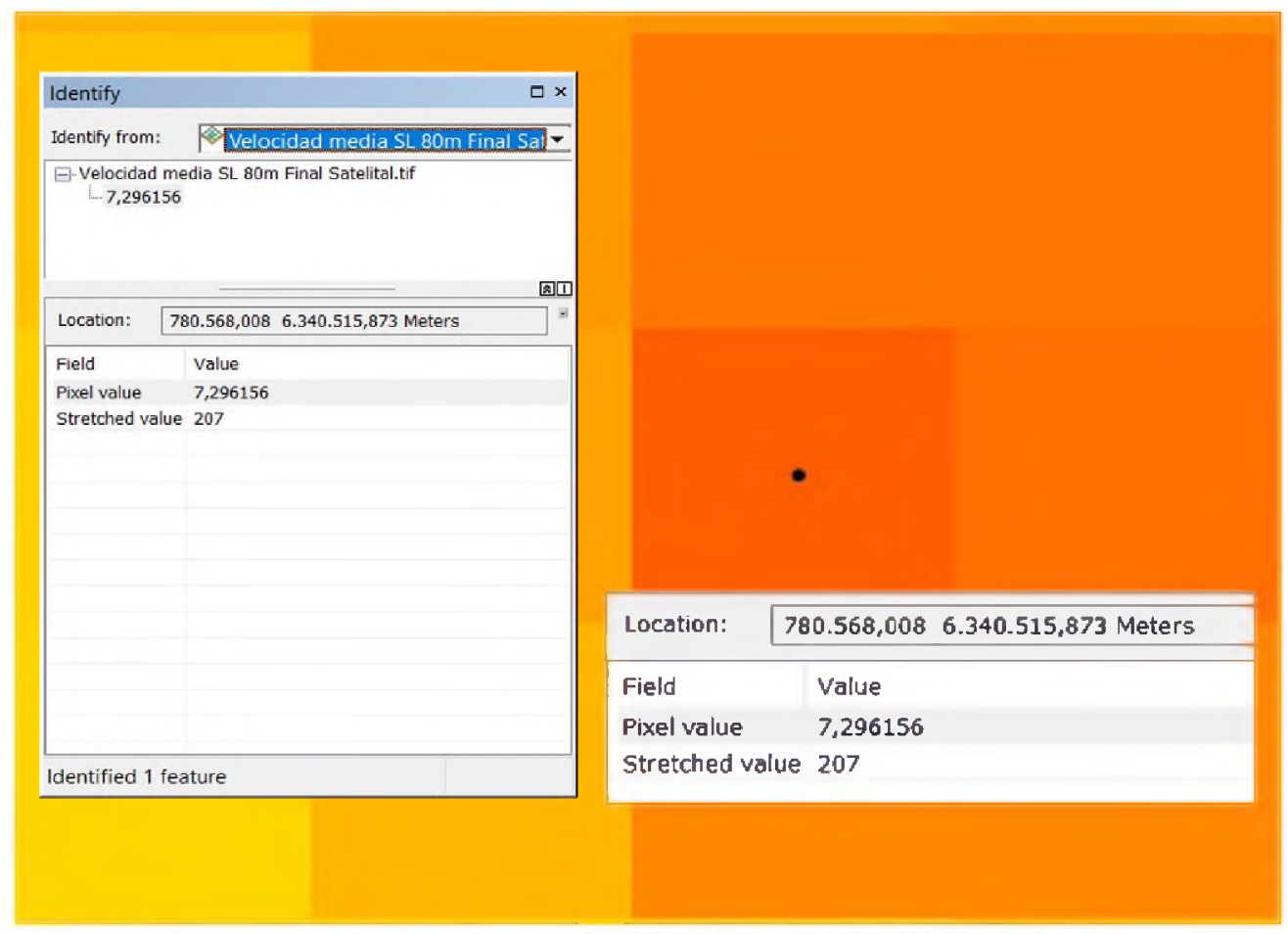

Figura 2: Punto geográfico y valor de la velocidad media del viento dado por una estación meteorológica determinada.

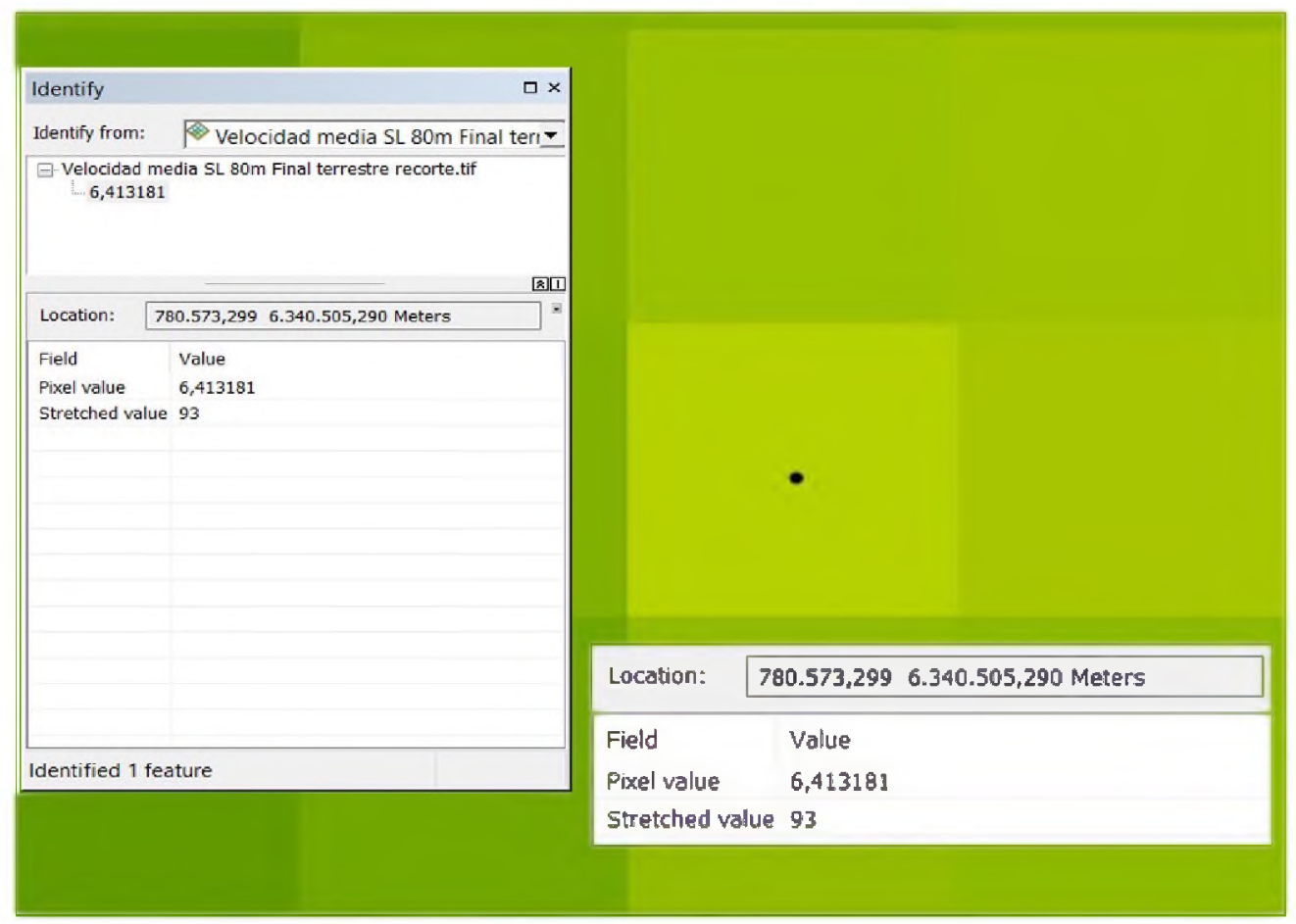

Figura3: Punto geográfico y valor de la velocidad media del viento, de igual coordenada del anterior, obtenido del MERRA-2. 
Como se mencionó anteriormente se decidió utilizar el software IBM-SPSS para análisis estadístico, procediendo de la siguiente manera:

\section{- Prueba t de Student}

Se aplicó la prueba $\mathrm{T}$ de Student para dos muestras Relacionadas.
Usado cuando es de interés comparar la velocidad media de vientos anuales usando la misma muestra, pero tomada en 2 circunstancias distintas.

Por lo que aquí interesa comparar las diferencias entre 2 variables (Terrestre y Satelital) numéricas de un mismo punto geográfico.

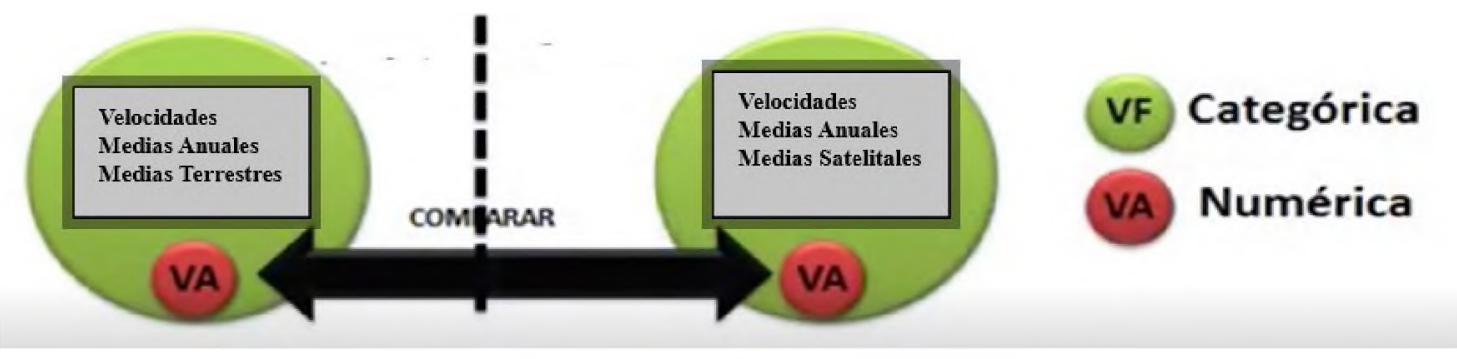

Figura 4: Característica de aplicación de "T" Student

\section{- Prueba de Hipótesis}

Se tomó 15 mediciones en los mismos puntos geográficos con el mismo historial y frecuencia, en diferentes lugares de los mapas eólicos en cuestión.

\section{- Hipótesis de la investigación}

Se planteó la hipótesis, en base a la siguiente pregunta: ¿Existe una diferencia significativa entre las medidas de Viento (velocidad y dirección) Terrestres y Satelitales? Resultando:

\section{Hipótesis Nula:}

$\mathrm{HO}=$ No hay diferencia significativa en las medias de las Velocidades Terrestres y las Velocidades Satelitales. Esta hipótesis es que no existen relación entre las variables.

\section{Hipótesis Alternativa:}

$\mathrm{H} 1=$ Hay una diferencia significativa en las medias de las Velocidades Terrestres y las Velocidades Satelitales.

Planteadas para un nivel de confianza del $99 \%(\alpha=0,01)$.

\begin{tabular}{|c|c|c|c|c|c|}
\hline & & \multicolumn{3}{|c|}{ PRUEBAS NO PARAMÉTRICAS } & $\begin{array}{c}\text { PRUEBAS } \\
\text { PARAMÉTRICAS }\end{array}$ \\
\hline \multicolumn{2}{|c|}{$\begin{array}{l}\text { Variable Aleatoria } \\
\text { Variable Fija }\end{array}$} & $\begin{array}{l}\text { NOMINAL } \\
\text { DICOTÓMICA }\end{array}$ & $\begin{array}{l}\text { NOMINAL } \\
\text { POLITÓMICA }\end{array}$ & ORDINAL & NUMÉRICA \\
\hline \multirow{3}{*}{$\begin{array}{l}\text { Estudio } \\
\text { Transwersal } \\
\text { Muestras } \\
\text { Independientes }\end{array}$} & Un grupo & $\begin{array}{c}\mathrm{x}^{2} \text { Bondad de Ajuste } \\
\text { Binomial }\end{array}$ & $\begin{array}{c}x^{2} \text { Bondad de } \\
\text { Ajuste }\end{array}$ & $\begin{array}{c}x^{2} \text { Bondad de } \\
\text { Aluste }\end{array}$ & $\begin{array}{l}\text { Tde student } \\
\text { (una muestra) }\end{array}$ \\
\hline & $\begin{array}{c}\text { Dos } \\
\text { erupos }\end{array}$ & $\begin{array}{l}\mathrm{x}^{2} \text { Bondad de Ajuste } \\
\text { Corrección de Yates } \\
\text { Test exacto de Fisher }\end{array}$ & $\begin{array}{c}x^{2} \text { de } \\
\text { Homozeneidad }\end{array}$ & $\begin{array}{l}\text { U Mann- } \\
\text { Withney }\end{array}$ & $\begin{array}{l}\text { Tde Student } \\
\text { (muestras } \\
\text { Independientes) }\end{array}$ \\
\hline & $\begin{array}{l}\text { Mass de } \\
\text { dos } \\
\text { prupos }\end{array}$ & $x^{2}$ Bondad de Ajuste & $\begin{array}{l}x^{2} \text { Bondad de } \\
\text { Ajuste }\end{array}$ & $\begin{array}{l}\text { H Kruskal- } \\
\text { Wallis }\end{array}$ & $\begin{array}{l}\text { ANOVA con un } \\
\text { factor } \\
\text { INIERsujetos }\end{array}$ \\
\hline \multirow{2}{*}{$\begin{array}{l}\text { Eatudio } \\
\text { Longitudinal } \\
\text { Muestras } \\
\text { Relacionadas }\end{array}$} & $\begin{array}{c}\text { Dos } \\
\text { medidas }\end{array}$ & Mc Nemar & Q de cochran & Wilcoxon & $\begin{array}{l}\text { Te student } \\
\text { (muestras } \\
\text { Reloxionodatio }\end{array}$ \\
\hline & $\begin{array}{l}\text { Mas de } \\
\text { dos } \\
\text { Medidas }\end{array}$ & Q de cochran & Q de cochran & Friedman & $\begin{array}{l}\text { ANOVA para medisks } \\
\text { repetidas } \\
\text { (ivirasuietoso) }\end{array}$ \\
\hline
\end{tabular}




\section{- Calculo del P-Valor}

Se calcula el p-valor, pero antes se verifica el supuesto de normalidad usando la prueba de Chapiro Wilk para muestras pequeñas menores a 30 , lo que debería dar un p-valor $\leq 0,01$.

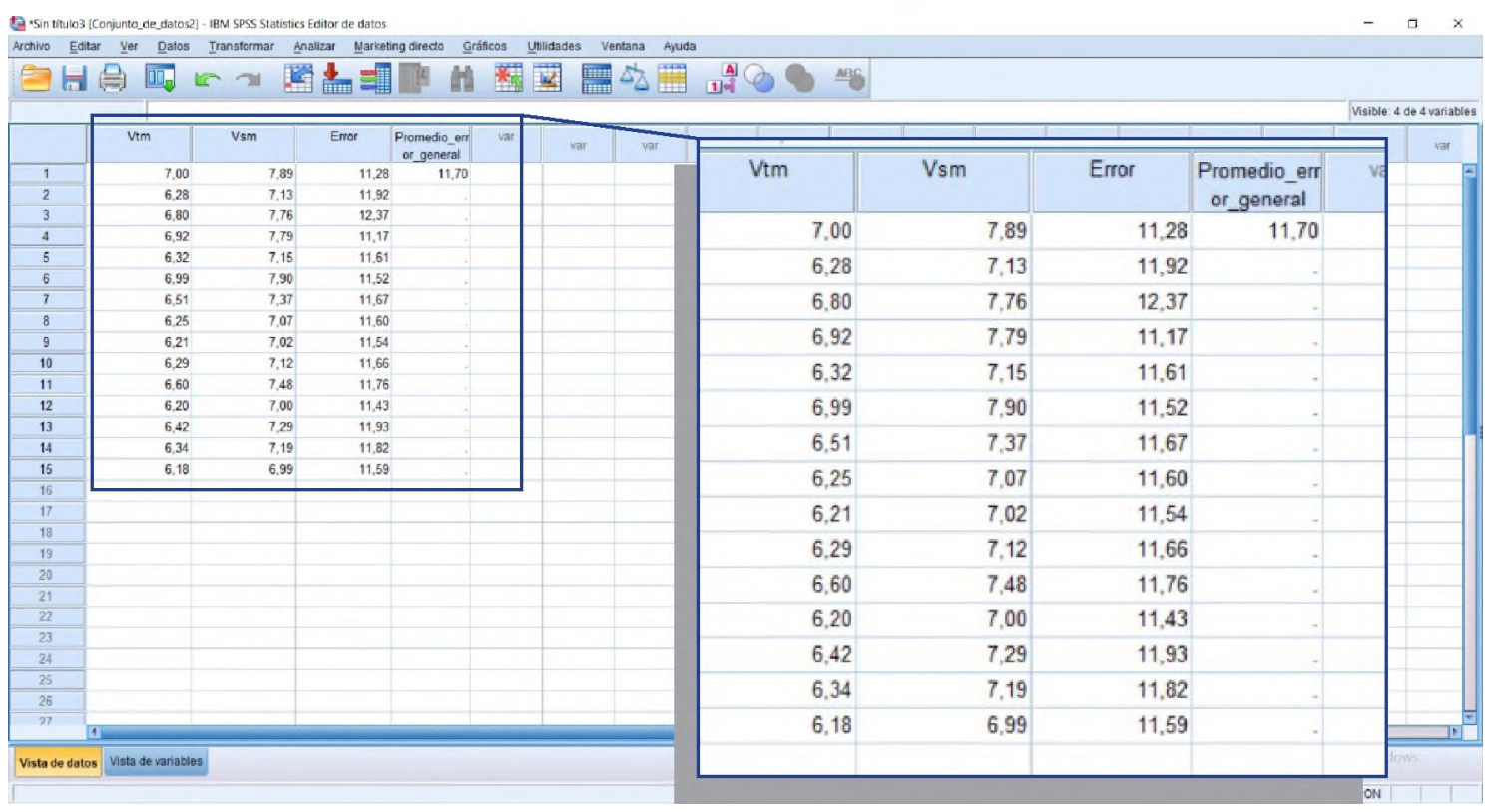

Figura 5: Resultados obtenidos del uso de software

Se cuenta con una base de datos de 15 media anual) respectivamente, con su difevalores numéricos de Vtm (Velocidad Terres- rencia porcentual entre ellas en ese mismo tre media anual) y Vsm (Velocidad Satelital punto y el error medio de todos sus valores.

\begin{tabular}{|c|c|c|}
\hline \multicolumn{3}{|l|}{ Explorar } \\
\hline \multicolumn{3}{|l|}{ Comentarios } \\
\hline Entrada & $\begin{array}{l}\text { Conjunto de datos activo } \\
\text { Filtro } \\
\text { Ponderación } \\
\text { Segmentar archivo } \\
\mathrm{N} \text { de filas en el archivo de } \\
\text { datos de trabajo }\end{array}$ & Conjunto de datos \\
\hline Manejo de valor perdido & $\begin{array}{l}\text { Definición de ausencia } \\
\text { Casos utilizados }\end{array}$ & $\begin{array}{l}\text { Los valores perdidos definidos por } \\
\text { el usuario para variables } \\
\text { dependientes se tratan como } \\
\text { perdidos. } \\
\text { Los estadisticos se basan en } \\
\text { casos sin valores perdidos para } \\
\text { ninguna de la variable dependiente } \\
\text { o factor utilizado. }\end{array}$ \\
\hline \multirow[t]{2}{*}{ Recursos } & Tiempo de procesador & 00:00:00,91 \\
\hline & Tiempo transcurrido & $00: 00: 00,84$ \\
\hline
\end{tabular}

Tabla 2: Nota generada por el software sobre su utilización 


\begin{tabular}{|c|c|c|c|c|c|c|}
\hline & \multicolumn{6}{|c|}{ Casos } \\
\hline & \multicolumn{2}{|c|}{ Válido } & \multicolumn{2}{|c|}{ Perdidos } & \multicolumn{2}{|c|}{ Total } \\
\hline & $\mathrm{N}$ & Porcentaje & $\mathrm{N}$ & Porcentaje & $\mathrm{N}$ & Porcentaie \\
\hline Terrestre & 15 & $100,0 \%$ & 0 & $0,0 \%$ & 15 & $100,0 \%$ \\
\hline Satelital & 15 & $100,0 \%$ & 0 & $0,0 \%$ & 15 & $100,0 \%$ \\
\hline
\end{tabular}

Tabla 3: Resumen del procesamiento de casos

\begin{tabular}{|c|c|c|c|c|c|c|}
\hline & \multicolumn{6}{|c|}{ Casos } \\
\hline & \multicolumn{2}{|c|}{ Válido } & \multicolumn{2}{|c|}{ Perdidos } & \multicolumn{2}{|c|}{ Total } \\
\hline & $\mathrm{N}$ & Porcentaie & $N$ & Porcentaie & $N$ & Porcentaje \\
\hline Terrestre & 15 & $100,0 \%$ & 0 & $0,0 \%$ & 15 & $100,0 \%$ \\
\hline Satelital & 15 & $100,0 \%$ & 0 & $0,0 \%$ & 15 & $100,0 \%$ \\
\hline
\end{tabular}

Tabla 4: Descriptivos

\begin{tabular}{|c|c|c|c|c|c|c|c|c|}
\hline & \multicolumn{7}{|c|}{ Percentiles } \\
\hline & & 5 & 10 & 25 & 50 & 75 & 90 & 95 \\
\hline \multirow{2}{*}{$\begin{array}{l}\text { Promedio } \\
\text { ponderado(Definición } \\
\text { 1) }\end{array}$} & Terrestre & 6,1800 & 6,1920 & 6,2500 & 6,3400 & 6,8000 & 6,9940 & \\
\hline & Satelital & & & & & & & \\
\hline \multirow[t]{2}{*}{ Bisagras de Tukey } & Terrestre & & & 6,2650 & 6,3400 & 6,7000 & & \\
\hline & Satelital & & & 7,0950 & 7,1900 & 7,6200 & & \\
\hline
\end{tabular}

Tabla 5: Percentiles

\begin{tabular}{|l|r|r|r|r|r|r|}
\hline & \multicolumn{3}{|c|}{ Kolmogorov-Smimov } & \multicolumn{3}{c|}{ Shapiro-Wilk } \\
\cline { 2 - 7 } & Estadistico & \multicolumn{1}{|c|}{ gl } & \multicolumn{1}{c|}{ Sig. } & Estadistico & gl & \multicolumn{1}{c|}{ Sig. } \\
\hline Terrestre & 222 & 15 & .046 &, 843 & 15 &, 014 \\
Satelital & 209 & 15 & .076 &, 851 & 15 & .018 \\
\hline
\end{tabular}

Tabla 6: Prueba de normalidad

Corrección de significación de Lilliefors

\section{Terrestre}

Terrestre Stem-and-Leaf Plot

Frequency Stem E Leaf

$\begin{array}{ll}9,00 & 6.122222334 \\ 5,00 & 6.56899 \\ 1,00 & 7.0\end{array}$

Stem width: 1,00

Each leaf: 1 case(s) 


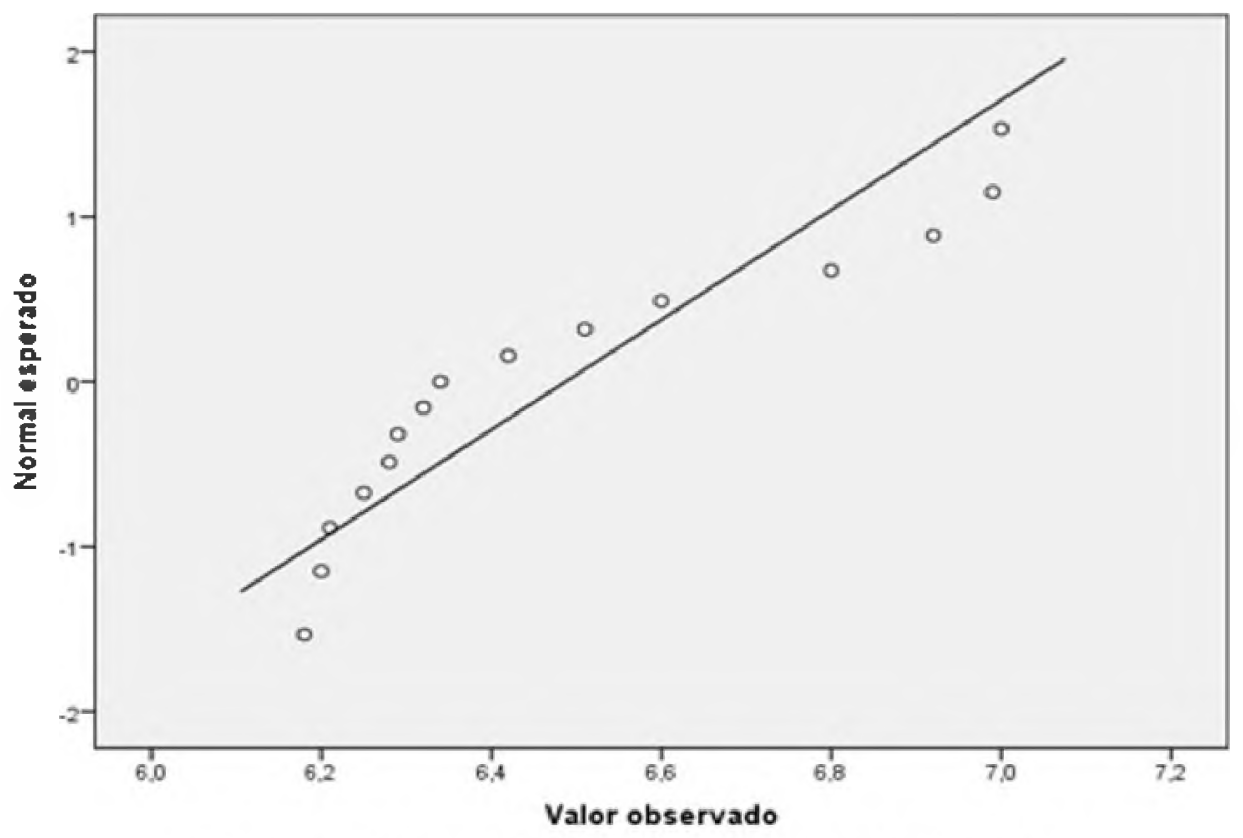

Figura 6: Grafico $Q-Q$ normal terrestre

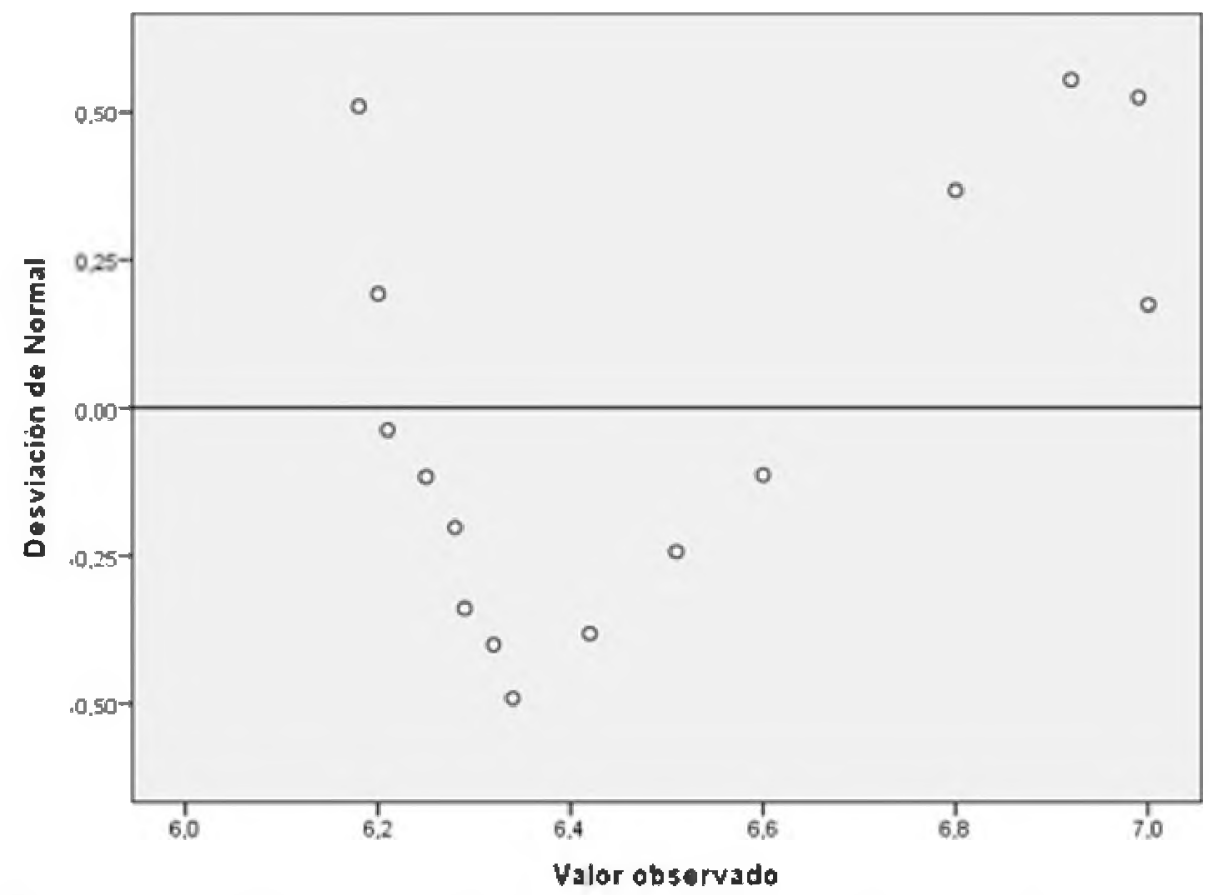

Figura 7: Grafico $Q-Q$ normal sin tendencia de terrestre 


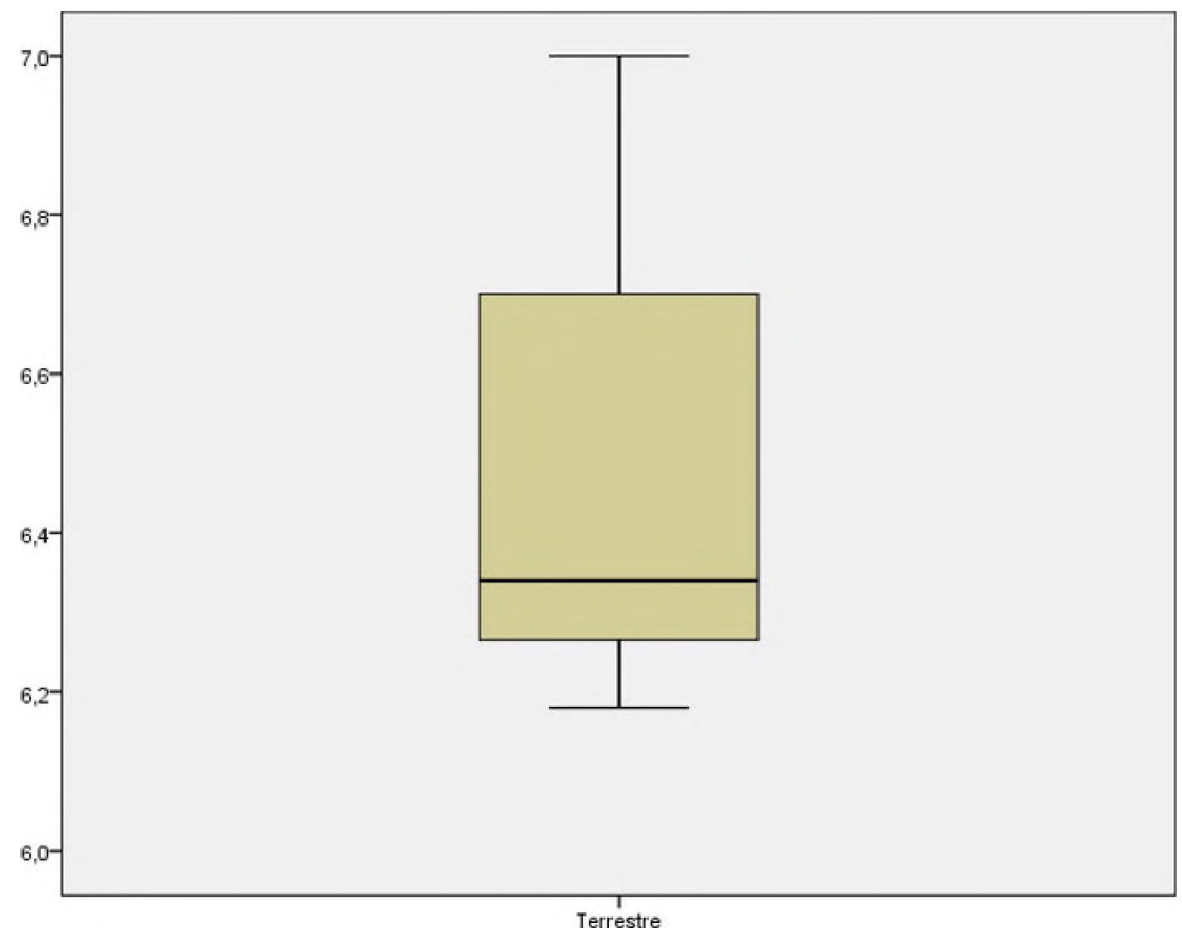

Figura 8: Grafico Caja y bigotes terrestre

\section{Satelital}

Satelital Stem-and-Leaf Plot

Frequency Stem \& Leaf

$1,00 \quad 6.9$

$10,00 \quad 7.0001111234$

$4,00 \quad 7.7789$

Stem width: 1,00

Each leaf: 1 case(s) 


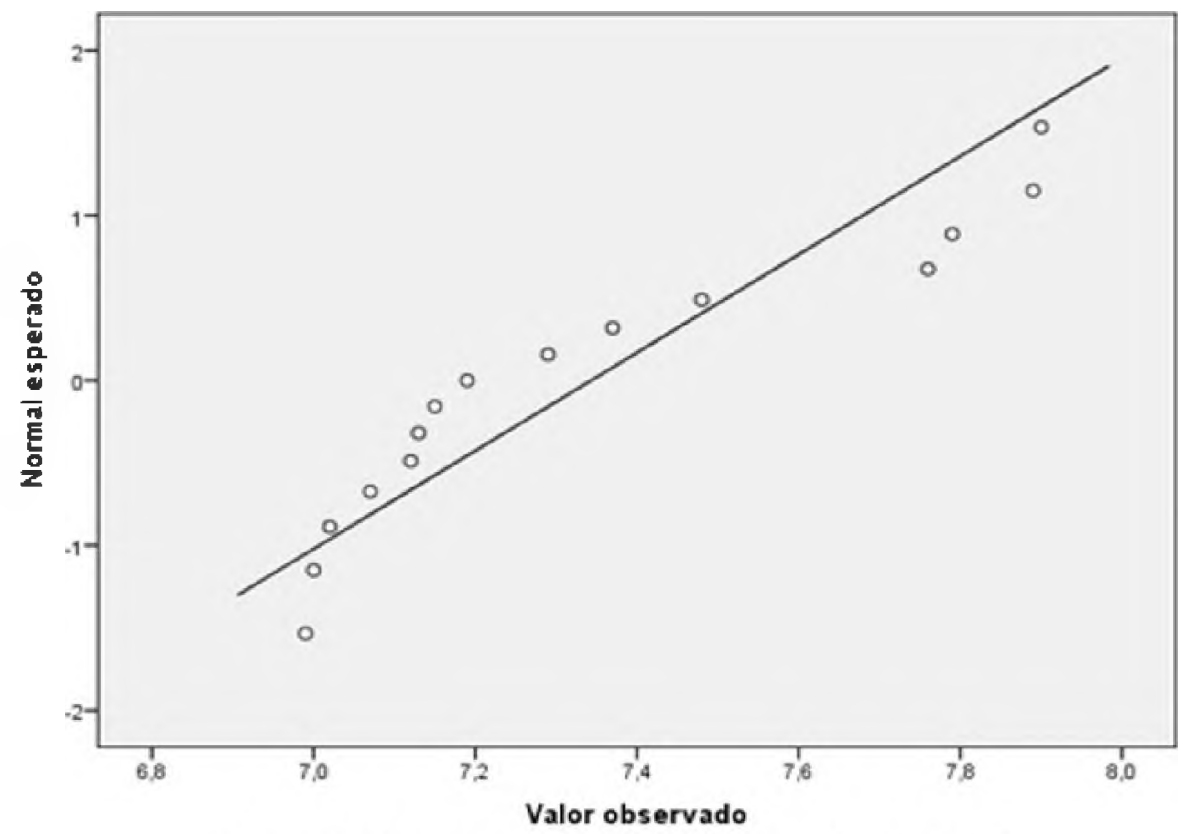

Figura 9: Grafico $Q-Q$ normal satelital

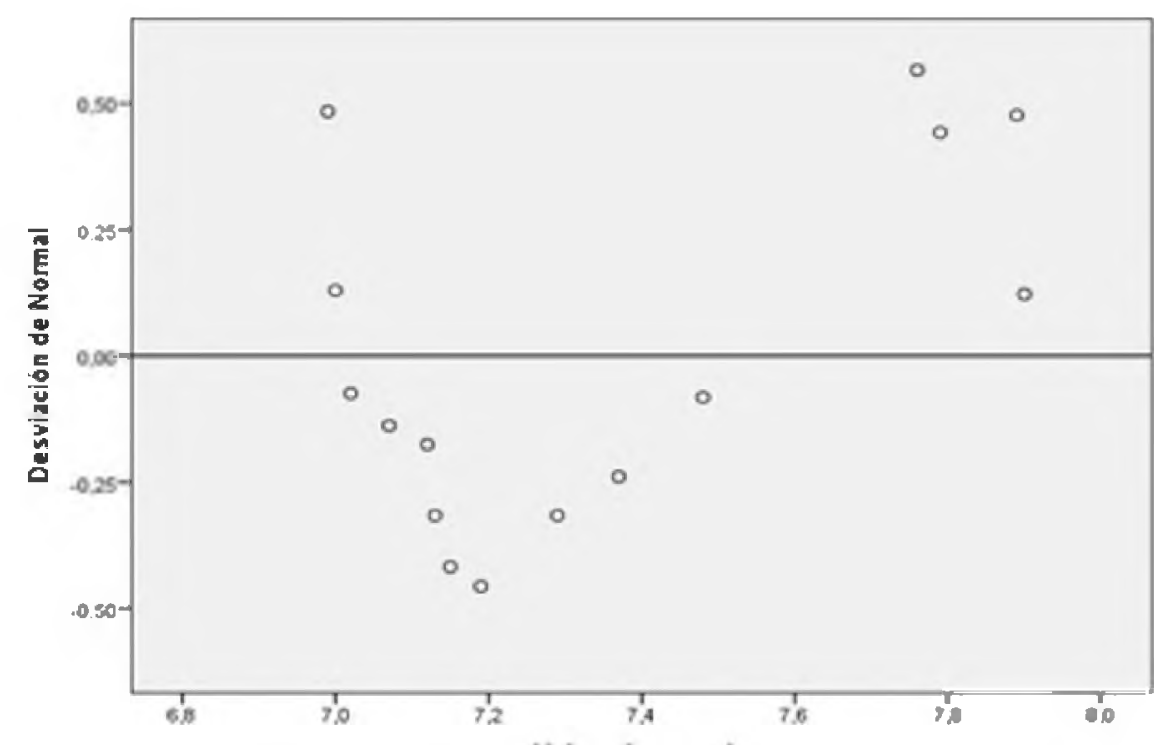

Figura 10: Grafico $Q-Q$ normal sin tendencia del satelital 


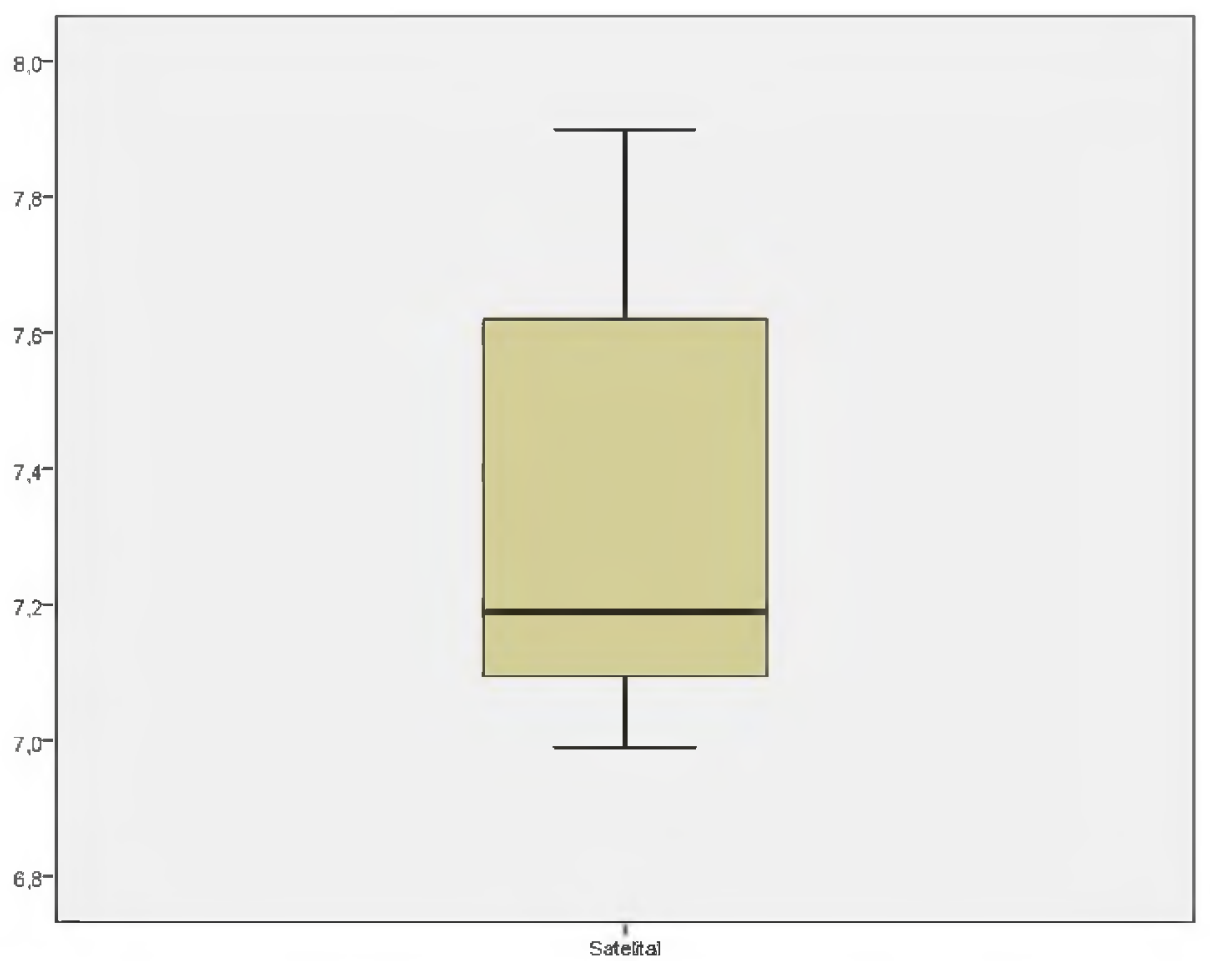

Figura 11: Grafico de caja y bigotes satelital

Prueba T

\begin{tabular}{|ll|c|r|r|c|}
\hline & Media & $\mathrm{N}$ & $\begin{array}{c}\text { Desviación } \\
\text { estándar }\end{array}$ & $\begin{array}{c}\text { Media de error } \\
\text { estándar }\end{array}$ \\
\hline Par 1 & Satelital & 7,3433 & 15 &, 33619 &, 08680 \\
& Terrestre & 6.4873 & 15 &, 30001 &, 07746 \\
\hline
\end{tabular}

Tabla 7: Estadística de muestras emparejadas

\begin{tabular}{|ll|r|r|r|}
\hline & N & Correlación & Sig. \\
\hline Par 1 Satelital \& Terrestre & & 15 & .997 & .000 \\
\hline
\end{tabular}

Tabla 8: Correlación de muestras emparejadas 


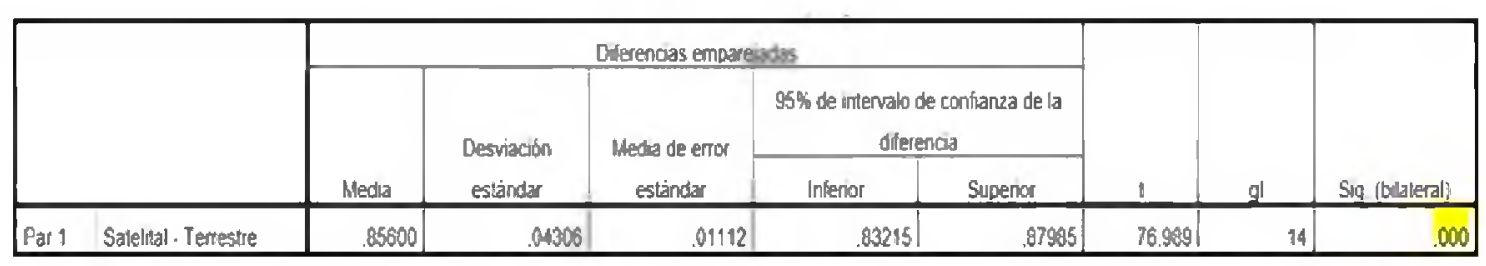

Tabla 9: Prueba de muestras emparejadas

En base a los resultados obtenidos anteriormente, se verifica si existe o no, una diferencia significativa entre Vtm y Vsm.

Se comprueba si se cumple el supuesto de normalidad, entre ellas. Empleando el software SPSS se obtuvo las gráficas con todos los datos validos en un 100\%, también los estadísticos descriptivos, las medias de
Vtm y Vsm, lo que evidencio una diferencia significativa. Usando el método de Chápiro Wilk para muestras pequeñas menores a 30 datos, donde los valores de la tabla son mayores al alfa $=0,01$, tomado, por lo que se acepta que la Hipótesis Nula H0 es $=0$ - mayor que 0,01 , es decir que provienen de una distribución normal.

\begin{tabular}{|l|r|r|r|r|r|r|}
\hline & \multicolumn{4}{|c|}{ Kolmogorov-Smimov } & \multicolumn{3}{c|}{ Shapiro-Wilk } \\
\cline { 2 - 7 } & Estadistico & \multicolumn{1}{|c|}{ gl } & \multicolumn{1}{c|}{ Sig. } & Esladistico & \multicolumn{1}{c|}{ gl } & \multicolumn{1}{c|}{ Sig. } \\
\hline Terrestre & .222 & 15 & .046 & .843 & 15 & .014 \\
Satelital & .209 & 15 & .076 & .851 & 15 & .018 \\
\hline
\end{tabular}

Tabla 10: Prueba de normalidad

Siguiendo con el analisis, realizamos el T Student en SPSS para 2 muestras relacionadas (Vtm; Vsm) los resultados son Muestras Estadisticos.

\begin{tabular}{|c|r|r|r|r|r|}
\hline & Media & $\mathrm{N}$ & \multicolumn{1}{c|}{$\begin{array}{c}\text { Desviación } \\
\text { estándar }\end{array}$} & $\begin{array}{c}\text { Media de error } \\
\text { estándar }\end{array}$ \\
\hline \multirow{2}{*}{ Par 1} & Satelital & 7,3433 & 15 & .33619 & .08680 \\
& Terrestre & 6,4873 & 15 & .30001 &, 07746 \\
\hline
\end{tabular}

Tabla 11: Estadistica de muestras emparejadas

Observo la media de Vtm y la media de Vsm y las velocidades medias Satelitales son mayores que las Terrestre, luego verifico si estás medidas son significativas o no? En la tabla de prueba para muestras relacionadas, se observa que la significancia es de "0,000
$\%$, por lo que es menor que $0,01 \%$ nuestro nivel alfa.

El criterio para decidir es:

Si la probabilidad obtenida de P-Valor es menor o igual a alfa, se rechaza $\mathrm{HO}$ (Se acepta H1) 
Si la probabilidad obtenida P-Valor es mayor a alfa, no rechazo $\mathrm{H} 0$, ( Se asepta $\mathrm{H} 0$ ).

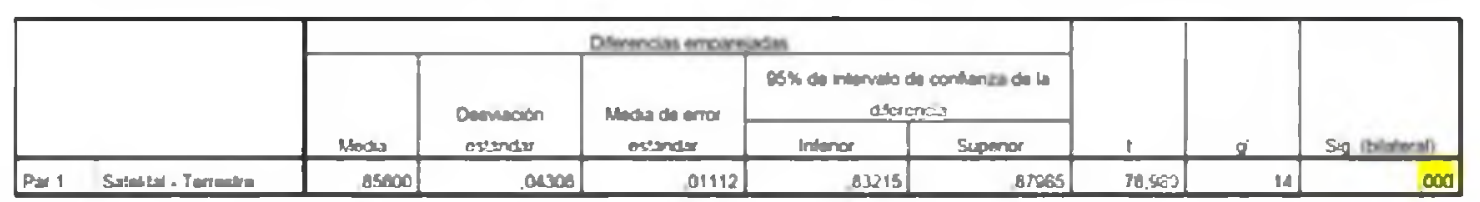

Tabla 12: Prueba de muestras emparejadas

\section{CONCLUSIONES}

Empleando tecnicas estadisticas para la comparacion de los datos medidos, se observo una diferencia significativa en las medias de los valores Vtm ( Velocidad del viento terrestre media) y Vsm ( velocidad del viento satelital media) lo que conduciria a mapas eolicos diferentes.

\begin{tabular}{|c|c|c|c|c|}
\hline & A & $\theta$ & c & [ \\
\hline 1 & BJECTI & $x$ & $\psi$ & Walor Mtax Madia S-T \\
\hline 2 & 1 & 674751.5313 & 6475194 EB & 6. $5415 \mathrm{~B} 5065$ \\
\hline 3 & 2 & 675054.2401 & $6475+946$ & 6. 9465.74409 \\
\hline 4 & 3 & 677158.9450 & EAT5104 & 6.955585957 \\
\hline 5 & 4 & B79.359,B5T & E4T.5194 198 & 6.9731699.27 \\
\hline 6 & 5 & 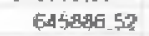 & 6473902.171 & 6. 979519117 \\
\hline 7 & 6 & 672366.1136 & 6473992.171 & $6.937213-21$ \\
\hline 日 & ? & $6735-49025$ & 6475942.171 & 6.936445001 \\
\hline 9 & 8 & $674751,531\}$ & 647392.171 & $6,93646257 ?$ \\
\hline 10 & 9 & 675954,2401 & 6473902,171 & $6,941805-471$ \\
\hline 11 & 10 & 677159,9489 & 6473992,171 & $6,954 B+4521$ \\
\hline 12 & 11 & 6.7E5599.65T & 6473992,171 & B.9754217 \\
\hline 13 & 12 & BPQ5A2 3065 & 64 T\$992, 171 & 8.909021126 \\
\hline 14 | & 13 & EPOJ65.0757 & 64 $3 \$ 02.171$ & 7.014517969 \\
\hline 15 & 14 & B8196h? TB41 & 64J3992.171 & T.016027927 \\
\hline 96 & 15 & $68 \$ 170,4929$ & 64 โ79ge? 171 & J प13]5 A8:45 \\
\hline
\end{tabular}

Con estos resultados y conociendo el error sistematico entre las medidas de los valores medios de Vtm y Vsm que es (1$0,883) / 2=0,0585$ se realizo un nuevo mapa resultante de la combinacion de ambas medias, lo que resulto en la siquiente imagen Raster.

En base a la hoja de calculo mostrada

\begin{tabular}{|c|c|c|c|c|}
\hline 17 & 16 & 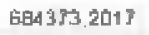 & 64) 3092.171 & ?.107gas \\
\hline 18 & 17 & 685575.9105 & 6473902.171 & 7.01310104 \\
\hline 19 & 18 & Geb378 BA193 & Bd 73492.171 & 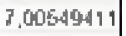 \\
\hline 20 & 19 & 689794, 3281 & 6473900.171 & 7,00029044 \\
\hline 21 & 20 & 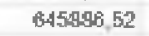 & 6472789.463 & 6,928569 \\
\hline 22 & 21 & B4 $70 \mathrm{LBg}$. 22RB & 6472789.463 & 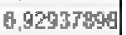 \\
\hline 23 & 22 & 648291. 9376 & 6472789.463 & 6. 9500550 \\
\hline 24 & 23 & B4,9404, 8464 & $6472 T: 49.463$ & 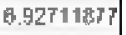 \\
\hline 25 & 24 & 650695,3552 & 6472789.463 & [5.9230004a \\
\hline 29 & 5 & 651900 DEA & 6472789.463 & 6.91ำ151견 \\
\hline 27 & 26 & 65\$1027 728 & 6472789.463 & 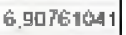 \\
\hline 29 & 27 & BS4 305,4016 & 6472789.443 & 6. \\
\hline 29 & 28 & 60940096 & E 4727040 & 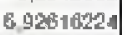 \\
\hline 30 & 29 & 6711 4⿻13 4048 & 6472789,463 & (9,9201309 \\
\hline 31 & 30 & 672746,11396 & 6472789.463 & 629014 \\
\hline 32 & 31 & 673548825 & 6472789.463 & 4,930BAs, \\
\hline
\end{tabular}

Tabla 13: Hoja para recalcular los pixeles usados en el nuevo mapa RASTER (52.422 datos)

en la tabla 13, se recalcularon los valores de pixel, y con la ayuda de un software GIS (Geographic Information System), Recordando que el mismo es una integración organizada de hardware, software y datos geográficos diseñada para capturar, almacenar, manipular, analizar y desplegar en todas sus formas la información geográficamente referenciada con el fin de resolver problemas complejos de planificación y de gestión; se realizo el nuevo mapa de extencion Raster.

El resultado del tratamiento anterior con el software GIS, puede verse en la figura 12, el cual permitio convalidar las medidas satelitales a las medidas terrestres, llegando a la conclusion de que se puede usar el mismo metodo conociendo unas pocas instalaciones confiables homologadas y de buena calidad para realizar mapas de recursos eolicos. 


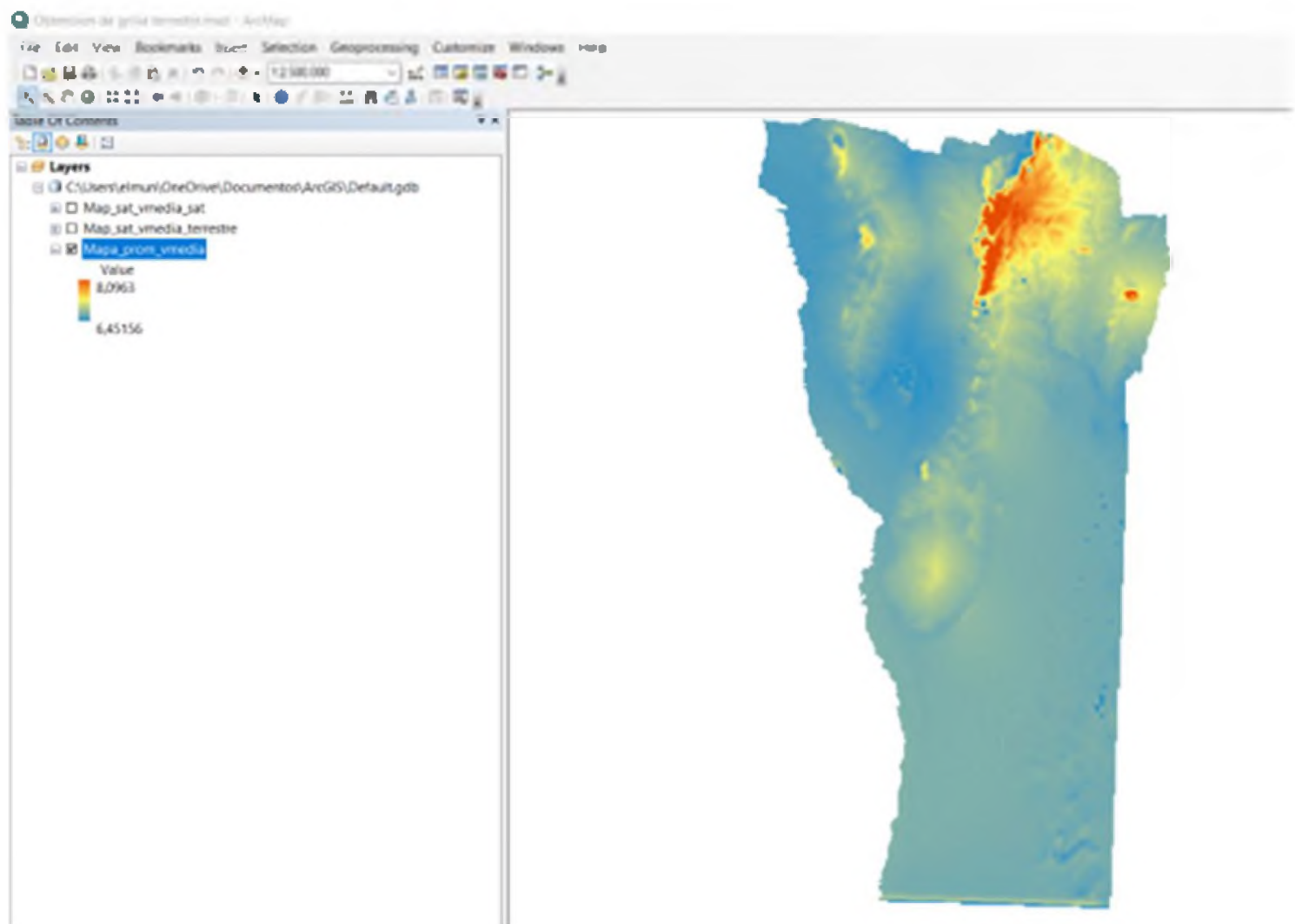

Figura 12: Imagen que nos permite convalidar las medidas satelitales a las medidas terrestres.

\section{REFERENCLAS}

[1]Medición, tratamiento y Análisis de Datos de Vientos Software WINDOGRAPHER DE HECTOR FERNANDO MATIOS C.R.E.E Centro Regional Energía Eólica

[2]Software Argentina-Map (Open Wind) C.R.E.E del Centro Regional Energía Eólica

[3]Software Global MapperV17 procesamiento de datos SIG (Sistema de Información Geográfica) con todo tipo de información cartográfica y de mapas para profesionales.

[4]Software IBM-SPSS Estadística

[5]Introducción a la Estadística autor Galindo Villardon

[6]Análisis y diseño de experimentos, Gutiérrez Pulido y De La Vara Salazar - 2a edic. $-2008$

[7]Arcgis 10.5 - Programa, Manual

[8]Artículos de Internet y videos varios de Internet 